\title{
A FAULT TOLERANT VEHICLE STABILITY CONTROL USING ADAPTIVE CONTROL ALLOCATION
}

\author{
Ozan Temiz \\ Department of Mechanical Engineering \\ Bilkent University \\ Bilkent, Ankara 06800 \\ Email: ozan.temiz@bilkent.edu.tr
}

\author{
Melih Cakmakci* \\ Yildiray Yildiz \\ Department of Mechanical Engineering \\ Bilkent University \\ Bilkent, Ankara 06800 \\ Turkey
}

\begin{abstract}
This paper presents an integrated fault-tolerant adaptive control allocation strategy for four wheel frive - four wheel steering ground vehicles to increase yaw stability. Conventionally, control of brakes, motors and steering angles are handled separately. In this study, these actuators are controlled simultaneously using an adaptive control allocation strategy. The overall structure consists of two steps: At the first level, virtual control input consisting of the desired traction force, the desired moment correction and the required lateral force correction to maintain driver's intention are calculated based on the driver's steering and throttle input and vehicle's side slip angle. Then, the allocation module determines the traction forces at each wheel, front steering angle correction and rear steering wheel angle, based on the virtual control input. Proposed strategy is validated using a non-linear three degree of freedom reduced two-track vehicle model and results demonstrate that the vehicle can successfully follow the reference motion while protecting yaw stability, even in the cases of device failure and changed road conditions.
\end{abstract}

\section{NOMENCLATURE}

$m$ Vehicle mass.

$J_{z}$ Vehicle moment of inertia about $z$ axis.

$L$ Overall length between front and rear axle.

$L_{f}$ Length between front axle and vehicle CoG.
$L_{r}$ Length between rear axle and vehicle CoG.

$A_{v}$ Frontal area of the vehicle.

$C_{d}$ Drag coefficient of the vehicle.

$\rho$ Density of the air.

$d$ Width of the wheelbase.

$C_{\alpha}$ Linear lateral friction coefficient.

$\alpha_{i}$ Lateral slip at the $i^{t h}$ tire, $i \in[1,2,3,4]$.

$N_{i}$ Normal force at the $i^{\text {th }}$ tire, $i \in[1,2,3,4]$.

$V$ Longitudinal velocity of vehicle.

$\beta$ Side slip angle of vehicle.

$r$ Yaw rate of vehicle.

$M$ Moment around $z$ axis of the vehicle.

$\delta_{1}$ Total front steering angle.

$\delta_{1 \text { in }}$ Front steering angle input.

$\Delta \delta_{1}$ Front steering angle correction.

$\delta_{2}$ Rear steering angle.

$F_{x i j}$ Net longitudinal force at $i j$ tire, $i \in[f, r], j \in[l, r]$.

$F_{y i j}$ Net lateral force at $i j$ tire, $i \in[f, r], j \in[l, r]$.

$F_{x i}$ Traction force acting on the $i^{\text {th }}$ tire, $i \in[1,2,3,4]$.

$F_{y i}$ Lateral force acting on the $i^{t h}$ tire, $i \in[1,2,3,4]$.

$A$ State matrix of linearized vehicle model

$B_{u}$ Input matrix of linearized vehicle model

$u_{s}$ Input vector of linearized vehicle model

$u$ Input vector used in adaptive control allocation

$F_{\text {in }}$ Desired total traction force.

$F$ Current total traction force.

$M_{c}$ Required total moment around $z$ axis.

${ }^{*}$ Address all correspondence to this author. 
$v$ Virtual control input vector.

$r_{\text {ref }}$ Reference yaw rate.

$A_{m}$ Reference model matrix of adaptive control allocation

\section{INTRODUCTION}

Over the past two decades, there have been many advancements in the automotive field with the increased interaction of vehicle subsystems that are traditionally designed separately. Vehicle communication networks, low-cost sensors and dependable mechatronic actuators play an important role in this new trend, which leads to designing the vehicle as a single mechatronic system, generating redundancies in control problems $[1,2]$.

One example of this redundancy can be seen when yaw rate control (i.e. regulation of rotation of the vehicle about the zaxis) in vehicle stability control is considered. Recently, electric in-wheel motors are getting popular which leads to an increased ability of traction control at each wheel. Using torque vectoring, traction controllers can regulate the yaw rate of the vehicle by changing the torque at each wheel. Four-wheel steering is another way of affecting the yaw rate in passenger vehicles. This technology is starting to become feasible due to dependable actuators at lower costs.

There are a few examples of control development in automotive literature that exploits the redundancy provided by alternative actuation methods. In [3], wheel cost minimization based integrated traction and four wheel steering control is proposed. More recently, in [4], Tavasoli proposed an optimization algorithm including rear wheel steering and brakes to achieve vehicle control objectives. In [5], a robust approach for fault tolerance in integrated control of traction forces and active steering is presented. Moreover in [6], presented system can switch between to different optimal control allocation schemes in order to compensate the fault. In [2], a concurrent controller structure that regulates both the energy management and traction control for parallel hybrids was proposed. An important challenge to exploit these redundancies is to find common approaches that will work in most vehicle problems and to overcome the computational complexity due to the increased number of objectives and constraints originating from the subsystem design problems.

Existence of two additional actuation methods besides the conventional front wheel steering to create yaw moment around vehicle presents an interesting but more complicated control allocation problem for automotive systems. Control allocation is a well-known concept that describes the methods to distribute the control effort among available actuators. One example of such methods is given for vehicle control in [7], where the approach presented in [8] is used. Typically, controllers feature allocation contain two sequential calculation steps. In the first step, the overall control effort is calculated using conventional control methods. This effort is then distributed to available actuators as the second step. A detailed survey of recent work about control allocation explaining various methodologies can be found in [9].

In this work, a novel vehicle stability control algorithm is developed by solving a yaw rate and vehicle acceleration feedback control problem first and then allocating the calculated controller effort to wheel and steering actuators by solving an adaptive control allocation problem. The allocation algorithm handles the force distribution among the wheels, and the steering composition between the front and the rear wheels. The required moment calculated in the first step and the desired traction force acts as virtual control input in order to follow yaw rate and force reference. Furthermore, in order to ensure stability of the vehicle depending on the side slip angle being larger than a threshold value a lateral force reference is generated. Front wheel steering angle is a composition of both an input from the driver and a correction command while the rear steering angle is completely generated by the algorithm.

The primary contribution of this paper can be given as a new adaptive control allocation method for vehicle stability control. Another adaptive control allocation method is presented for vehicle control in [8], [7]. It will be shown in the next sections of this paper that this approach is useful for the cases where vehicle dynamics parameters vary during operation and/or device failures.

In the next section of this paper, the mathematical model assuming a three-degree of freedom reduced two-track vehicle configuration equipped with front and rear steering capability is presented. In Section III, the components of the controller structure are discussed. Then, in Section IV, the developed controller is validated using simulations based on the non-linear model developed in Section II. Our initial conclusions and future work are discussed in Section V.

\section{MATHEMATICAL MODEL}

The vehicle stability controller developed in this paper features two primary methods of actuation, four-wheel traction and four-wheel steering. In modern vehicles, acceleration and orientation of the vehicle can be measured using inertial measurement units. In order to use during the controller development process, a modified version of the reduced non-linear two track model based on [10] is developed. The schematic representation for the two-track model can be seen in Fig. 1 explaining the forces acting on the vehicle and the related geometrical relationships. The variables that describe the states of the vehicle model are assumed as the vehicle speed, $V$, the side slip angle, $\beta$, and the yaw rate, $r$. The dynamic equations describing the motion of the vehicle can be developed by using the physical relationships shown in Fig. 1.

The acceleration of the vehicle can be calculated using the total force acting on the system in the current motion direction 


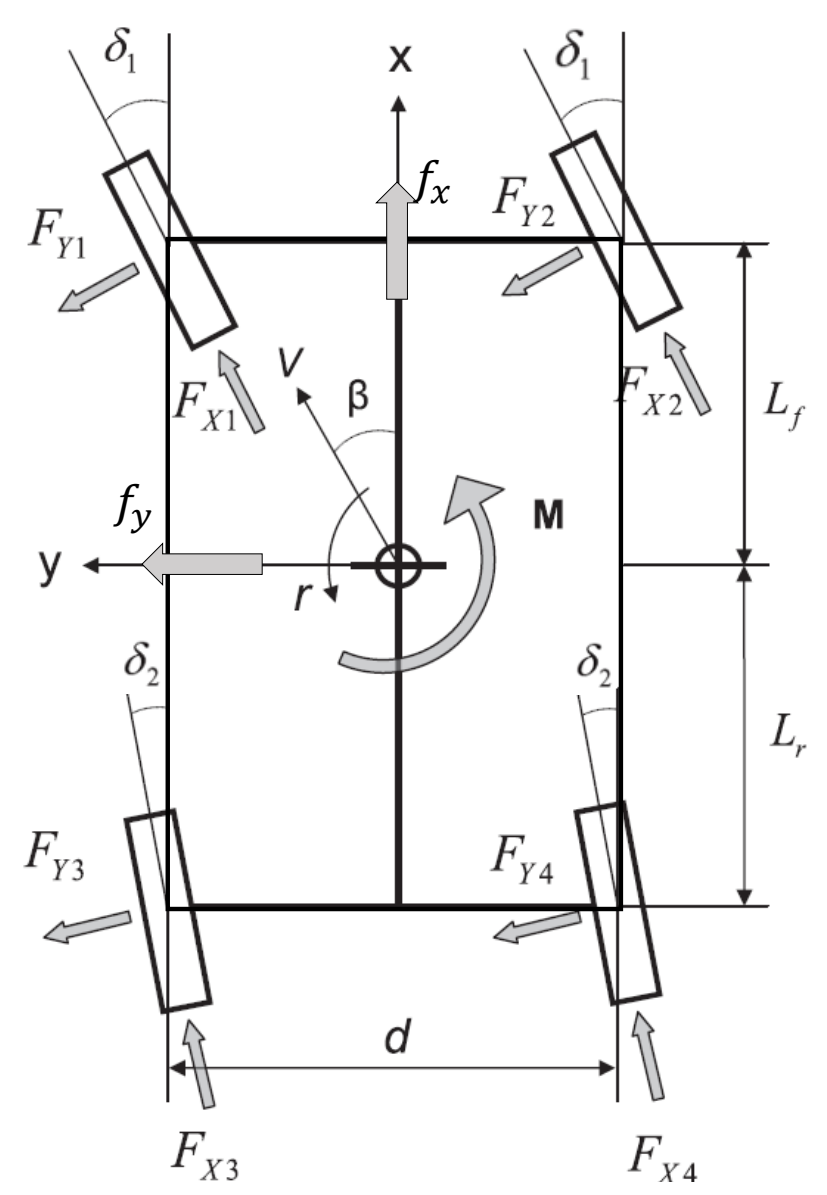

FIGURE 1: SCHEMATIC FOR MODIFIED TWO TRACK MODEL

and the inertia as shown in (1).

$$
\dot{V}=\frac{1}{m}\left[f_{x} \cos (\beta)+f_{y} \sin (\beta)-\frac{1}{2} C_{d} A_{v} \rho V^{2}\right]
$$

The total rate of change of the side slip angle, $\beta$ of the vehicle can be calculated using the total linear acceleration in the slip direction and the yaw rate as shown in (2).

$$
\dot{\beta}=\frac{1}{m V}\left[f_{x} \sin (\beta)+f_{y} \cos (\beta)\right]-r
$$

where $f_{x}, f_{y}$ are the net forces acting on the vehicle in $\mathrm{x}$ and $\mathrm{y}$ directions, and $M$ is the yaw moment.

Based on the diagram given in Fig. 1 the net forces on the vehicle in $\mathrm{x}$ - and $\mathrm{y}$ - directions can be calculated as shown in (3) and (4).

$$
\begin{aligned}
f_{x} & =\sum_{i=\{f, r\}} \sum_{j=\{l, r\}} F_{x i j} \\
f_{y} & =\sum_{i=\{f, r\}} \sum_{j=\{l, r\}} F_{y i j}
\end{aligned}
$$

The contribution to (3) and (4) from each wheel can be calculated using the force acting on each wheel and the steering angles, $\delta_{1}$ and $\delta_{2}$ for front and rear wheels respectively as shown in (5).

$$
\begin{aligned}
F_{x f l} & =F_{X 1} \cos \delta_{1}-F_{Y 1} \sin \delta_{1} \\
F_{y f l} & =F_{Y 1} \cos \delta_{1}+F_{X 1} \sin \delta_{1} \\
F_{x f r} & =F_{X 2} \cos \delta_{1}-F_{Y 2} \sin \delta_{1} \\
F_{y f r} & =F_{Y 2} \cos \delta_{1}+F_{X 2} \sin \delta_{1} \\
F_{x r l} & =F_{X 3} \cos \delta_{2}-F_{Y 3} \sin \delta_{2} \\
F_{y r l} & =F_{Y 3} \cos \delta_{2}+F_{X 3} \sin \delta_{2} \\
F_{x r r} & =F_{X 4} \cos \delta_{2}-F_{Y 4} \sin \delta_{2} \\
F_{y r r} & =F_{Y 4} \cos \delta_{2}+F_{X 4} \sin \delta_{2}
\end{aligned}
$$

Derivative of the yaw rate, $\dot{r}$, can be calculated using the total yaw moment on the vehicle and the inertia, $J_{z}$.

$$
\dot{r}=\frac{1}{J_{z}}[M]
$$

where total moment, $M$, generated by the wheel forces are calculated using the equation given in (7).

$M=\frac{d}{2}\left(F_{x f r}+F_{x r r}-F_{x f l}-F_{x r l}\right)+\left(F_{y f l}+F_{y f r}\right) L_{f}-\left(F_{y r l}+F_{y r r}\right) L_{r}$

Longitudinal and lateral tire forces are calculated based on the slip ratios $\alpha_{i}$, depending on the front tires (given in (8)) and rear tires ( given in (9)).

$$
\begin{aligned}
& \alpha_{1,2}=\delta_{1}-\beta-\frac{L_{f} r}{V} \\
& \alpha_{3,4}=\delta_{2}-\beta+\frac{L_{r} r}{V}
\end{aligned}
$$

When the slip angles are small, lateral forces can be calculated using the linear relationship given in (10).

$$
F_{Y i}=C_{\alpha} \alpha_{i} N_{i}
$$


where $C_{\alpha}$ is the linearized lateral friction coefficient and $N_{i}$ is the normal force acting on the $i^{\text {th }}$ tire which is calculated by using vehicle geometry and moment balance around the vehicle. Assuming that center of gravity of the vehicle lies at $\frac{d}{2}$, normal force calculation can be given as shown in (11).

$$
\begin{aligned}
& N_{f}=\frac{m g L_{r}}{2 L} \\
& N_{f}=\frac{m g L_{f}}{2 L}
\end{aligned}
$$

The longitudinal tire forces, $F_{X i}$, are determined as part of the control allocation algorithm. The effect of longitudinal slip dynamics is neglected. Using equations (8) - (10) and incorporating small angle assumptions, expressions in (5) can be linearized as shown in (12).

$$
\begin{aligned}
F_{x f l} & =F_{x 1} \\
F_{y f l} & =C_{\alpha} \alpha_{1} N_{f} \\
F_{x f r} & =F_{x 2} \\
F_{y f r} & =C_{\alpha} \alpha_{2} N_{f} \\
F_{x r l} & =F_{x 3} \\
F_{y r l} & =C_{\alpha} \alpha_{3} N_{r} \\
F_{x r r} & =F_{x 4} \\
F_{y r r} & =C_{\alpha} \alpha_{4} N_{r}
\end{aligned}
$$

Modified reduced two track nonlinear model given in (1)(12) can be linearized around a constant velocity , $V_{0}$, by assuming small side slip and steering angles. Combining (1) - (6), and assuming that the center of gravity is located at $\frac{d}{2}$ (i.e. $N_{1}=N_{2}$ and $N_{3}=N_{4}$ ), a linear state space representation of the reduced two track model can be obtained as

$$
\dot{x}=A x+B_{u} u_{s}
$$

where, $A, B_{u}, x$ and $u$ are given as

$$
\begin{gathered}
A=\left[\begin{array}{cccc}
0 & 0 & 0 \\
0 & -\frac{C_{\alpha}}{m V_{0}}\left(2 N_{f}+2 N_{r}\right) & \frac{C_{\alpha}}{m V_{0}^{2}}\left(2 L_{r} N_{r}-2 L_{f} N_{f}\right)-1 \\
0 & -\frac{C_{\alpha}}{J_{z}}\left(2 L_{r} N_{r}-2 L_{f} N_{f}\right) & -\frac{C_{\alpha}}{J_{z} V_{0}}\left(2 L_{r}^{2} N_{r}-2 L_{f}^{2} N_{f}\right)
\end{array}\right] \\
B_{u}=\left[\begin{array}{cccccc}
0 & 0 & \frac{1}{m} & \frac{1}{m} & \frac{1}{m} & \frac{1}{m} \\
-\frac{C_{\alpha} 2 N_{f}}{m V_{0}} & -\frac{C_{\alpha} 2 N_{r}}{m V_{0}} & 0 & 0 & 0 & 0 \\
\frac{C_{\alpha} 2 N_{f}}{J_{z}} & -\frac{C_{\alpha} 2 N_{r}}{J_{z}} & -\frac{d}{2 J_{z}} & \frac{d}{2 J_{z}} & -\frac{d}{2 J_{z}} & \frac{d}{2 J_{z}}
\end{array}\right]
\end{gathered}
$$

$$
\begin{aligned}
& x^{T}=\left[\begin{array}{lll}
V & \beta
\end{array}\right] \\
& u_{s}^{T}=\left[\begin{array}{llllll}
\delta_{1} & \delta_{2} & F_{x 1} & F_{x 2} & F_{x 3} & F_{x 4}
\end{array}\right]
\end{aligned}
$$

\section{CONTROL STRUCTURE}

The closed loop control structure used in this paper is presented in Fig. 2. Driver initiates motion by specifying a front steering input, $\delta_{1}$, and a desired traction force, $F_{i n}$, by manipulating the steering wheel and the pedals respectively.

In the Virtual Control Input Generation stage, desired traction force, $F_{c}$, the desired correction moment $M_{c}$ and the required lateral force correction $F_{y c}$ is generated depending on the driver inputs and measured vehicle states. Then, based on the virtual control input, the adaptive control allocation algorithm determines the traction force $F_{X i}$ applied at each wheel, rear wheel steering angle $\delta_{2}$ and front wheel steering angle correction $\Delta \delta_{1}$.

The control algorithm presented in this section is developed utilizing the linearized vehicle model given in (13) - (19), and the full non-linear model presented in (1) - (5) is used in the simulations shown in the next section.

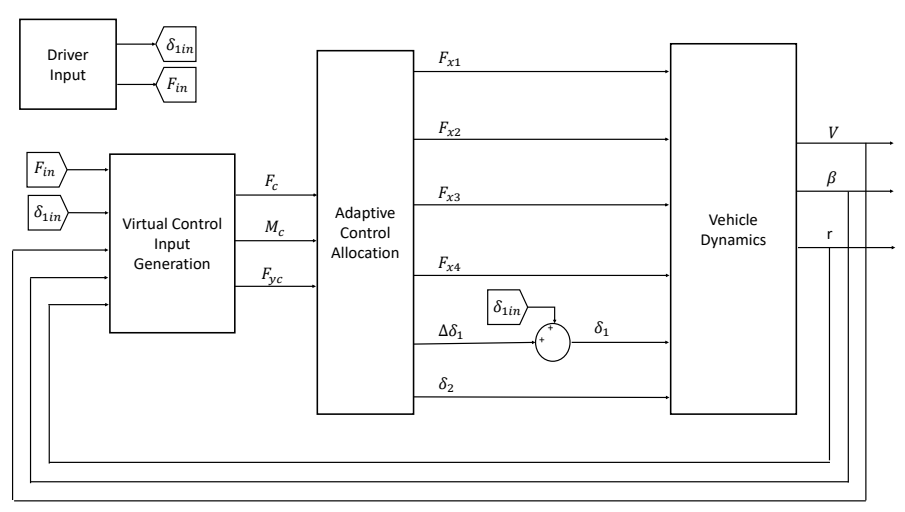

FIGURE 2: CONTROL STRUCTURE

\section{Virtual Control Input Generation}

In order to determine the virtual control input elements, required traction force, $F_{c}$, yaw rate correction moment $M_{c}$ and lateral force correction $F_{y c}$ should be calculated.

For traction force, force input from driver $F_{i n}$ and the current total traction force $F$ is used. In order to ensure that there is no steady-state error and vehicle is maintaining the driver's acceleration demand a PI scheme is used. Defining deviation from the desired value as $\tilde{F}=F_{\text {in }}-F$, the updated value of the desired traction force is as shown (18).

$$
F_{c}=K_{i} \int \tilde{F}+K_{p} \tilde{F} d t
$$


In order to calculate the desired yaw rate moment, $M_{c}$, first a desired yaw rate, $r_{\text {ref }}$ should be defined for the vehicle based on steering input and its current forward velocity. Reference yaw rate, $r_{r e f}$, is generated based on the approach presented in many vehicle dynamics literature such as [1], [11] as shown in (19).

$$
\begin{gathered}
r_{r e f}=\delta_{1 i n} \frac{V}{L+K_{u s} V^{2}} \\
K_{u s}=\frac{m L_{r}}{L C_{\alpha} N_{f}}-\frac{m L_{f}}{L C_{\alpha} N_{r}}
\end{gathered}
$$

Defining the deviation from the reference value as $\tilde{r}=r-r_{r e f}$, a PI controller is designed for moment command calculation to ensure that there will be no steady state error as shown in (20).

$$
M_{c}=K_{p} \tilde{r}+K_{i} \int \tilde{r} d t
$$

Lastly, in order to ensure stability of the vehicle while following yaw reference signal, lateral force correction is provided to the adaptive allocation system. To assure stability of the vehicle, side-slip angle, $\beta$, should be in the stable region as discussed in [7]. Since side-slip is directly related to lateral velocity of the vehicle, growth of the side-slip prevented by applying lateral force in the negative direction. Assuming that the side-slip angle of the vehicle can be estimated or measured, following simple law can be used [1].

$$
\begin{aligned}
& \text { If }|\beta| \geq \beta_{\text {threshold and } \beta . \Delta \beta>0} \\
& \text { Then } F_{y c}=-K_{p} \beta-K_{d} \dot{\beta}
\end{aligned}
$$
follows

Resulting virtual control input vector for this application as

$$
v^{T}=\left[\begin{array}{lll}
F_{c} & F_{y c} & M_{c}
\end{array}\right]
$$

\section{Adaptive Control Allocation}

The adaptive control allocation method used in this work is based on $[12,13]$ and the block diagram for this algorithm is presented in Fig. 3. The method is briefly explained below and the details can be found in [12].

Writing the actuator input vector as $u_{s}=u+\Delta u$, where

$$
\begin{aligned}
\Delta u & =\left[\begin{array}{llllll}
\delta_{1 \text { in }} & 0 & 0 & 0 & 0 & 0
\end{array}\right] \\
u & =\left[\begin{array}{lllllll}
\Delta \delta_{1} & \delta_{2} & F_{x 1} & F_{x 2} & F_{x 3} & F_{x 4}
\end{array}\right]
\end{aligned}
$$

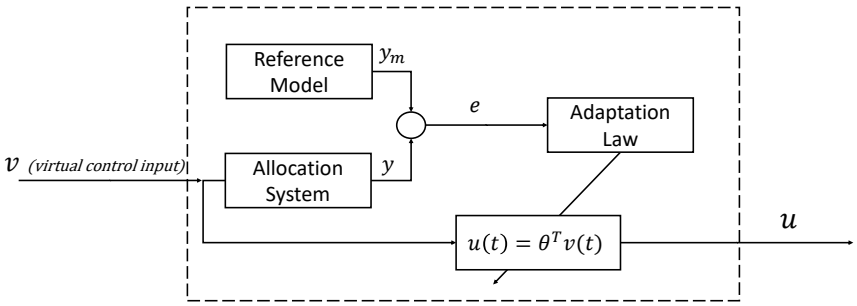

FIGURE 3: BLOCK DIAGRAM OF EXPLOITED ADAPTIVE CONTROL ALLOCATION

and decomposing the input matrix $B_{u} \in R^{3 x 6}$ as $B_{u}=B_{v} B$, where $B_{v}$ is a $3 \times 3$ matrix and $B$ is a $3 x 6$ matrix, (13) - (17) can be written as

$$
\begin{aligned}
\dot{x} & =A x+B_{u} u+B_{u} \Delta u \\
& =A x+B_{v} B u+B_{u} \Delta u
\end{aligned}
$$

To introduce actuator effectiveness uncertainty, a diagonal positive definite matrix $\Lambda \in R^{6 x 6}$ is added to (24), which leads to the following plant equations

$$
\begin{aligned}
\dot{x} & =A x+B_{v} B \Lambda u+B_{u} \Delta u \\
& =A x+B_{v} v+B_{u} \Delta u
\end{aligned}
$$

where $v \in R^{3}$ is virtual control input. The objective of the control allocation is to determine the actuator input vector $u$ such that

$$
B \Lambda u=v
$$

Consider the following dynamics

$$
\dot{y}=A_{m} y+B \Lambda u-v,
$$

where $A_{m} \in R^{3 \times 3}$ is stable, and a reference model

$$
\dot{y}=A_{m} y_{m} .
$$

The goal is to make the state vector $y$ follow the reference model output $y_{m}$. Determining the actuator input vector as

$$
u=\theta_{v}^{T} v
$$


where $\theta \in R^{3 x 6}$ is an adaptive parameter matrix and substituting (29) into (27), it is obtained that

$$
\dot{y}=A_{m} y+\left(B \Lambda \theta_{v}^{T}-I\right) \nu
$$

An ideal solution for the adaptive parameter vector is assumed to exist and satisfy $B \Lambda \theta_{v}^{T}=I$. Defining $\theta_{v}^{T}=\theta_{v}^{* T}-\tilde{\theta}_{v}^{T}$, where $\tilde{\theta}_{v}^{T}$ is the deviation of the adaptive parameter vector $\theta_{v}^{T}$ from its ideal value $\theta_{v}^{* T},(30)$ can rewritten as

$$
\dot{y}=A_{m} y+B \Lambda \tilde{\theta}_{v}^{T} v
$$

Defining the tracking error as $e=y-y_{m}$ and using (28)-(31), it is obtained that

$$
\dot{e}=A_{m} e+\Lambda \tilde{\theta}_{v}^{T} v
$$

Using an adaptation rate matrix $\Gamma=\Gamma^{T}=\gamma I_{r} \in R^{r x r}>0$, where $\gamma$ is a positive scalar and $I_{r}$ is an identity matrix, it can be shown [12] that the following adaptive law

$$
\dot{\theta}=\Gamma \operatorname{Proj}\left(\theta_{v},-v e^{T} P B\right)
$$

where Proj is the projection operator [14], leads to a stable adaptive control allocation. It is noted that $P$ in (33) is the positive definite symmetric solution of $A_{m}^{T} P+P A_{m}=-Q$ where $Q$ is a positive definite symmetric matrix.

The adaptive control allocation algorithm proposed in [12], and described above, is used to realize the virtual control input vector $v$ in (26), consisting of the total traction force, the lateral force correction, and the moment correction, by determining the actuator input vector $u$, whose elements are the front steering angle correction, the rear steering angle and the individual traction forces.

By using the linear equations of motion given in (13) - (17), the $B$ matrix can be calculated. Each column of the $B$ matrix corresponds to one of the allocated actuators while each row addresses one component of the $v$ vector:

- For the first row of the matrix $B$, the first two elements corresponds to steering inputs. Since in the linearized version of the vehicle model steering has no effect on traction forces these elements are selected as zero. Furthermore, because in typical driving conditions equal distribution of traction forces at each tire is desired, the last four elements of first row are selected as ones.

- For the second row, the first two elements are determined by calculating the effect of steering on lateral forces. The last four elements on this row are selected as zeros since the effect of traction forces on the lateral force is neglected due to the linearization.

- For the third row, the contribution of steering angles on the moment $M$ is calculated by using the lateral forces created due to steering angles, and the first two elements are created. Additionally the effect of the traction forces on the moment $M$ is shown by setting the therefore last four elements of the second row as $d / 2$.

The resulting $B$ is given as

$$
B=\left[\begin{array}{cccccc}
0 & 0 & 1 & 1 & 1 & 1 \\
C_{\alpha}\left(N_{1}+N_{2}\right) & C_{\alpha}\left(N_{3}+N_{4}\right) & 0 & 0 & 0 & 0 \\
C_{\alpha} L_{f}\left(N_{1}+N_{2}\right) & -C_{\alpha} L_{r}\left(N_{3}+N_{4}\right) & -\frac{d}{2} & \frac{d}{2} & -\frac{d}{2} & \frac{d}{2}
\end{array}\right]
$$

Therefore the $B_{v}$ matrix which satisfies the decomposition $B=B_{v} B$ can be calculated as

$$
B_{v}=\left[\begin{array}{ccc}
\frac{1}{m} & 0 & 0 \\
0 & \frac{-1}{m V_{0}} & 0 \\
0 & 0 & \frac{1}{J_{z}}
\end{array}\right]
$$

\section{SIMULATIONS}

A MATLAB/Simulink model is developed based on the system given in Fig. 2 using the nonlinear dynamic model from Section II. In order to evaluate the functionality and performance of the proposed control method, an object avoidance maneuver is simulated with different faults. Results are compared with a baseline vehicle control system where rear wheels are adjusted proportional to driver's front steering wheel input. Traction forces at the baseline vehicle are distributed evenly based on driver's acceleration request.

\section{Baseline Performance}

In order to understand the forthcoming results better the baseline performance of controller is first examined when there is no fault in the vehicle. Simulations are executed with a one period of sinusoidal steering input which simulates an object avoidance maneuver. Fig. 4, shows the simulation variables from the vehicle and the controller during the simulation. It can be seen that at this vehicle speed both systems performs satisfactorily following commands. the proposed controller causes more deceleration. On the other hand it follows yaw reference signal slightly better. The reason for that is introduction of more steering wheel in order to follow yaw rate reference.

When the forward velocity is higher, it is harder to safely rotate the vehicle. When the same steering maneuver is applied to both systems when the velocity is $20 \mathrm{~m} / \mathrm{s}$ baseline system fails. When the trajectory is examined both cars can make the first turn 

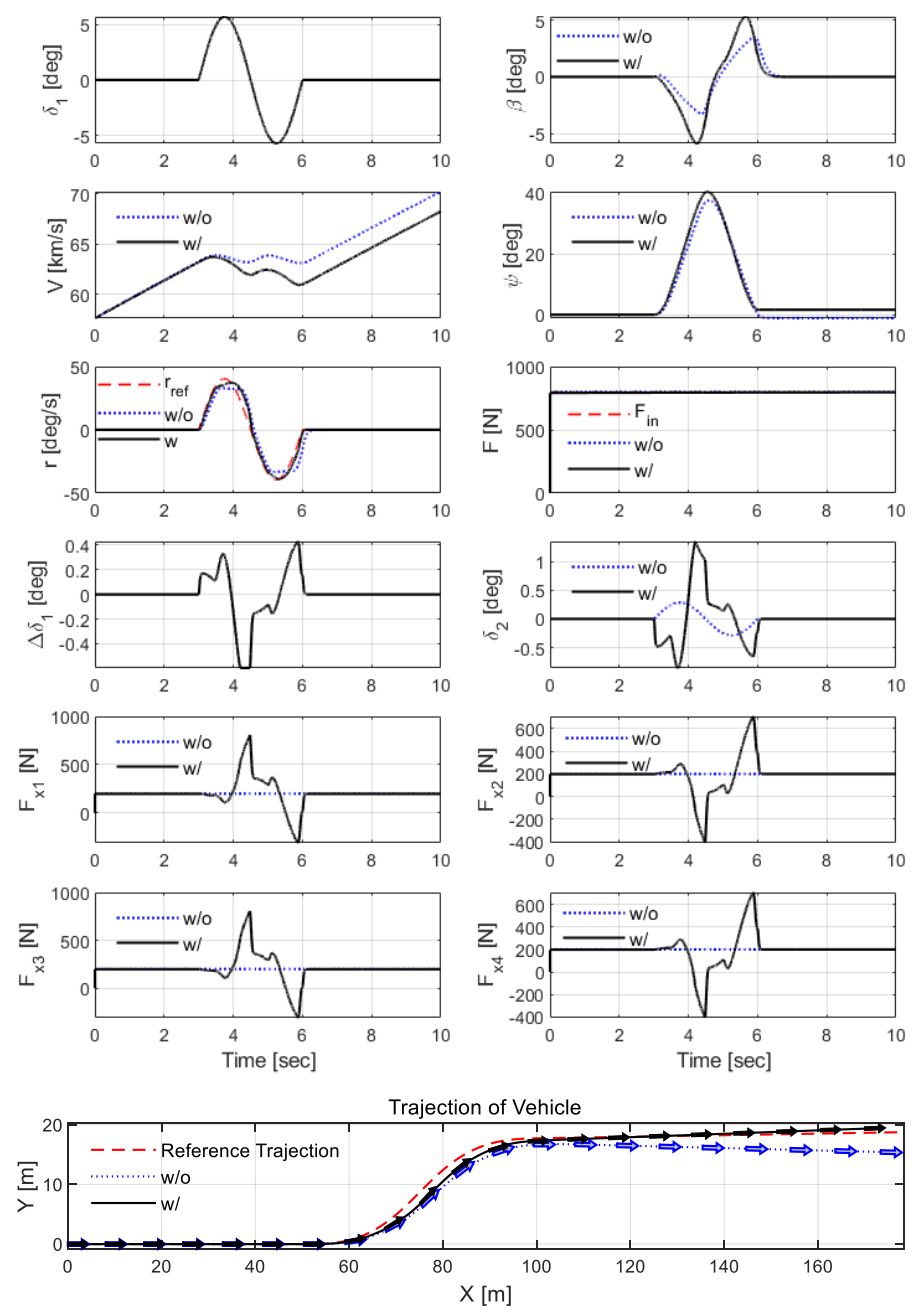

FIGURE 4: OBJECT AVOIDANCE MANEUVER WHEN $\mathrm{V}=16 \mathrm{~m} / \mathrm{s}$

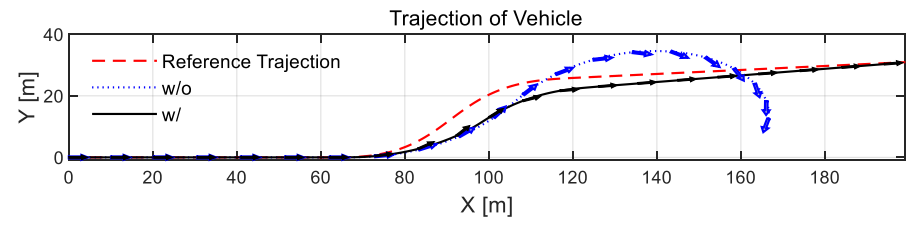

FIGURE 5: OBJECT AVOIDANCE MANEUVER TRAJECTORY WHEN V=20 m/s

however once vehicles have the lateral velocities without torque vectoring, and required RWS and FWS corrections baseline system cannot turn properly due to over steering and it fails. Fig. 5 .
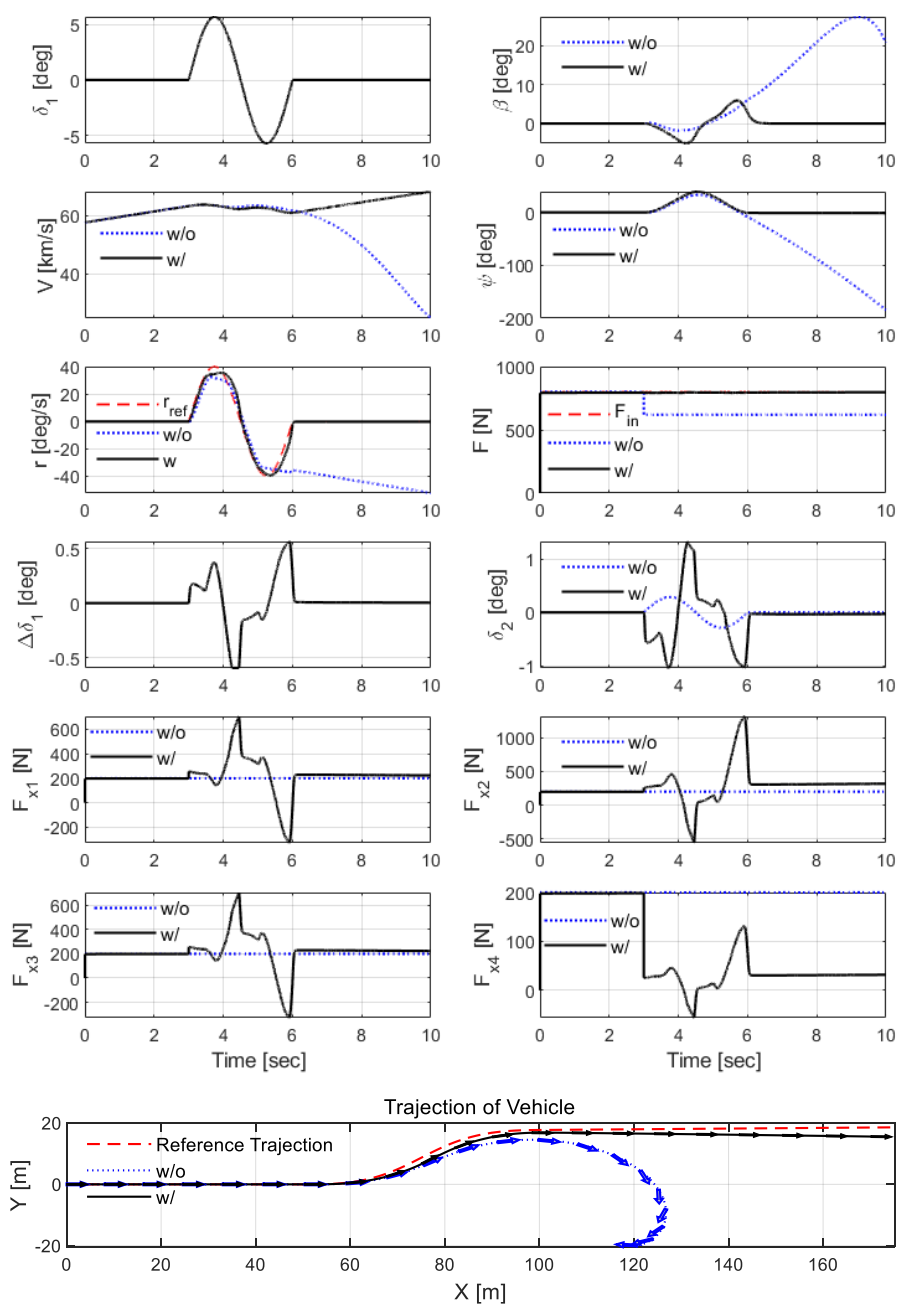

FIGURE 6: OBJECT AVOIDANCE MANEUVER WITH ACTUATOR FAILURE

\section{Actuator Failure}

To test the effectiveness of the proposed controller in emergency situations a scenario involving an actuator failure is considered. The rear right traction force reduced to $10 \%$ at $t=3 \mathrm{~s}$ which is also the start of the steering for the object avoidance maneuver. Results of the simulation can be seen at Fig. 6 When the results are examined, it can be seen that the yaw rate reference signal is successfully followed by the vehicle equipped with the new controller. Moreover, the traction forces at functioning wheels are increased to maintain desired acceleration while the front and the rear steering are used to compensate the moment due to uneven traction forces. Lastly since the rear right motor has failure vehicle tends turn right when the fault is not compensated. Therefore baseline system turn right much more than desired. As a result, it cannot follow the reference trajectory. 

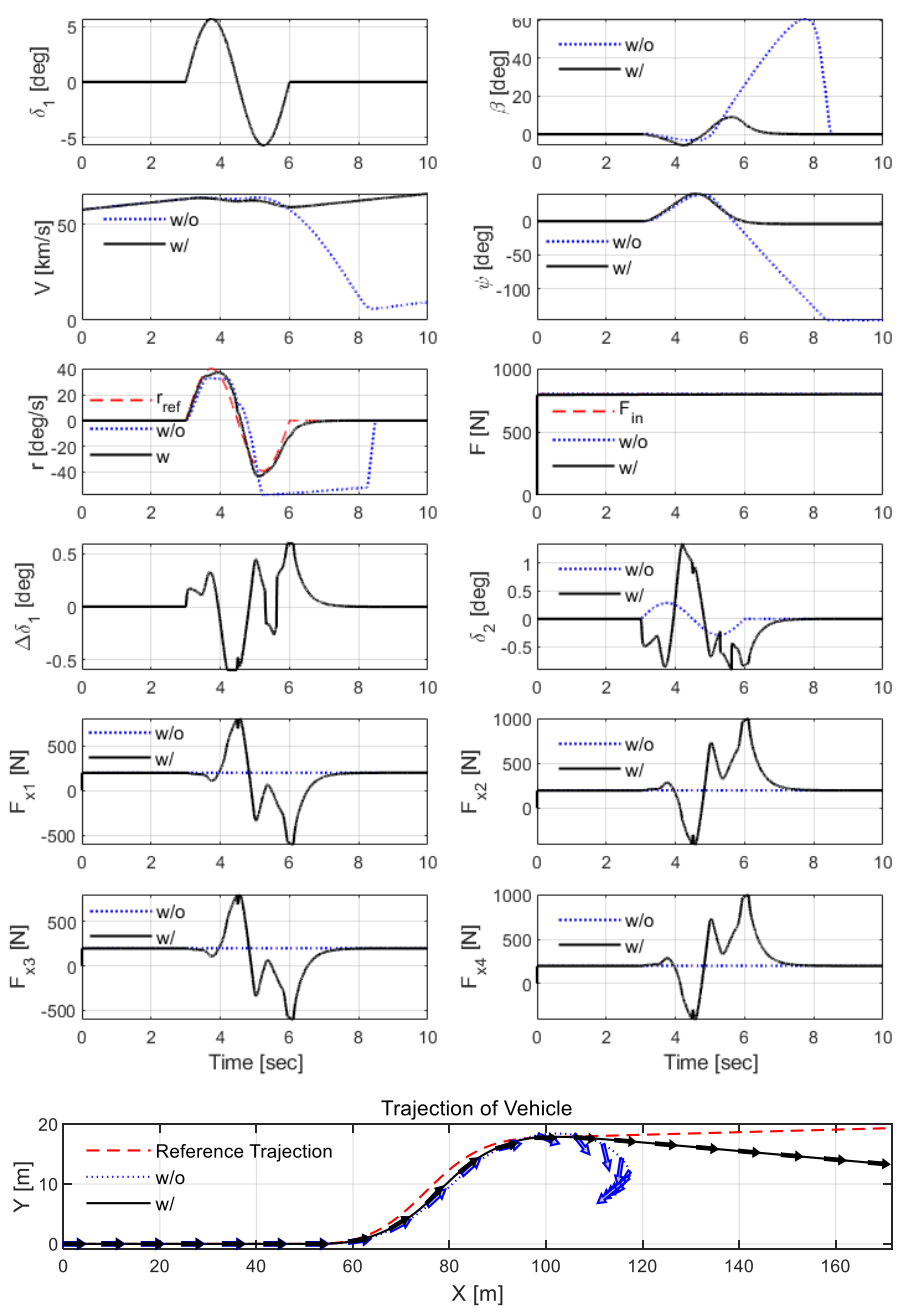

FIGURE 7: OBJECT AVOIDANCE MANEUVER WITH VARYING ROAD CONDITIONS

\section{Varying Road Conditions}

To emulate another type of an emergency condition and study the effectiveness of the proposed controller, the road surface conditions were used. In this simulation the road friction coefficient $C_{\alpha}$ for the right tires reduced to half of its value in order to simulate slippery road conditions starting at $t=4.5 \mathrm{~s}$ while the vehicle is commanded to perform the same object avoidance maneuver. The results in Fig. 7 show that the baseline vehicle had difficulty following the commands while the proposed controller performed as designed. In order to compensate varying road conditions, proposed system sends much more traction force to the right wheels. As a result it can follow the reference yaw signal. Moreover, trajectories, side slip angles and velocities show that vehicle equipped with the new controller remained stable while the baseline system failed, slipped and eventually stopped.
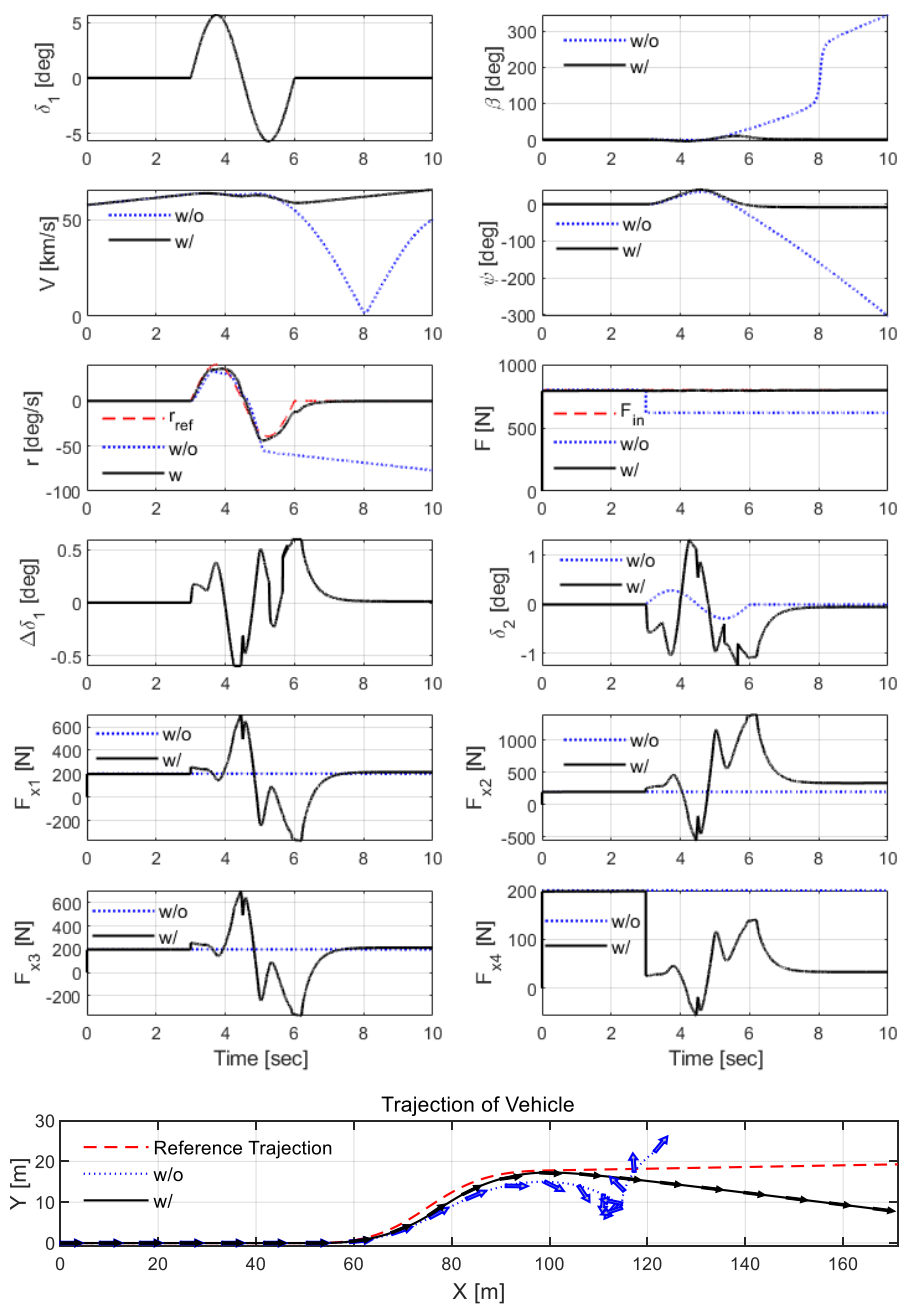

FIGURE 8: OBJECT AVOIDANCE MANEUVER WITH ACTUATOR FAILURE AND VARYING ROAD CONDITIONS

\section{Actuator Failure and Varying Road Conditions}

As a last step in our validation, a combined case when the traction force at rear right tire is reduced to $10 \%$ at $t=3$ and the road friction coefficient $C_{\alpha}$ for the rear tires reduced to half at $t=4.5 s$ during same steering maneuver is considered. Simulation results for this scenario is given in Fig. 8. Since both the failure and the variation occurs at the right, the baseline vehicle tends to turn right. Therefore vehicle spins when the steering input is towards right at the baseline system. However, for the vehicle with the new controller variations are compensated by torque vectoring and larger steering inputs. As a result vehicle is able to follow desired trajectory with no failure. 


\section{CONCLUSIONS}

In this paper an adaptive control allocation method for vehicle stability control is proposed. The controller is developed based on the linearized version of reduced two track model and simulations are performed on non-linear version of reduced two track model. Results from the simulations show that proposed controller performs better and safer than proportionally controlled baseline four-wheel drive and steering systems. It is also shown in simulations that the proposed control scheme can compensate failures that occur at the vehicle or variations in the environment during driving. For the future work proposed controller will be validated by using more complex model which uses nonlinear tire forces coupled with lateral tire dynamics. Furthermore, the inclusion of a nonlinear model to the adaptive allocation formulation and more fidelity in the vehicle dynamics are planned as the future work on this topic.

\section{ACKNOWLEDGMENT}

The authors would like to thank Seyed Shahabaldin Tohidi for his valuable input.

\section{REFERENCES}

[1] A. Galip Ulsoy, Huei Peng, and Melih Çakmakci. Automotive Control Systems. Cambridge University Press, 2012.

[2] H I Dokuyucu and M Cakmakci. Concurrent Design of Energy Management and Vehicle Traction Supervisory Control Algorithms for Parallel Hybrid Electric Vehicles. IEEE Transactions on Vehicular Technology, 65(2):555565, 2016.

[3] E. Ono, Y. Hattori, Y. Muragishi, and K. Koibuchi. Vehicle dynamics integrated control for four-wheel-distributed steering and four-wheel-distributed traction/braking systems. Vehicle System Dynamics, 44(2):139-151, 2006.

[4] Ali Tavasoli, Mahyar Naraghi, and Heman Shakeri. Optimized coordination of brakes and active steering for a $4 \mathrm{WS}$ passenger car. ISA Transactions, 51(5):573-583, sep 2012.

[5] Rongrong Wang, Hui Zhang, and Junmin Wang. Linear parameter-varying controller design for four-wheel independently actuated electric ground vehicles with active steering systems. IEEE Transactions on Control Systems Technology, 22(4):1281-1296, 2014.

[6] Yang Wang, Guohai Liu, Duo Zhang, Huawei Zhou, Hao Ye, and Xufang Chen. Combined Fault-Tolerant Control with Optimal Control Allocation for Four-Wheel Independently Driven Electric Vehicles. In 2016 IEEE Vehicle Power and Propulsion Conference (VPPC), pages 1-5. IEEE, oct 2016.

[7] Johannes Tjonnas and Tor A. Johansen. Stabilization of Automotive Vehicles Using Active Steering and Adaptive
Brake Control Allocation. IEEE Transactions on Control Systems Technology, 18(3):545-558, may 2010.

[8] Johannes Tjønnås and Tor Arne Johansen. Adaptive control allocation. Automatica, 44(11):2754-2765, nov 2008.

[9] Tor A. Johansen and Thor I. Fossen. Control allocation - A survey. Automatica, 49(5):1087-1103, 2013.

[10] Uwe Kiencke and Lars Nielsen. Automotive control systems: For engine, driveline, and vehicle: Second edition. Springer, 2005.

[11] R Rajamani. Vehicle Dynamics and Control. Springer, 2006.

[12] Seyed Shahabaldin Tohidi, Yildiray Yildiz, and Ilya Kolmanovsky. Fault tolerant control for over-actuated systems: An adaptive correction approach. Proceedings of the American Control Conference, 2016-July:2530-2535, 2016.

[13] S. S. Tohidi, Y. Yildiz, and I. Kolmanovsky. Adaptive Control Allocation for Over-Actuated Systems with Actuator Saturation. IFAC-PapersOnLine, 50(1):5492-5497, 2017.

[14] Eugene Lavretsky and Travis E. Gibson. Projection Operator in Adaptive Systems. 2011. 